\title{
Monitoring and Control System of the ATLAS RPCs in view of Run-2
}

\author{
Alessandro Polini ${ }^{a}$, on behalf of the ATLAS Collaboration \\ ${ }^{a}$ INFN Bologna, \\ via Irnerio 48, 40128 Bologna, Italy \\ E-mail: alessandro.polini@bo.infn.it
}

\begin{abstract}
Resistive Plate Chambers (RPCs) provide the barrel region of the ATLAS detector with an independent muon trigger and a two-coordinate measurement. The chambers, 3600 gas volumes in total covering a surface area of about $4000 \mathrm{~m}^{2}$, are arranged in three concentric double layers and operated in a strong magnetic toroidal field. During Run-1 the LHC has provided protonproton collisions at 7 and $8 \mathrm{TeV}$ in the centre-of-mass frame with a steady increase in instantaneous luminosity, summing up to an integrated luminosity of about $30 \mathrm{fb}^{-1}$. For Run-2, a further increase in beam energy, luminosity and also backgrounds is expected with rates and doses approaching the limits for which the RPCs were certified to work at irradiation facilities. In this scenario, a precise and granular monitoring of the detector (gas gap currents, gas flow, trigger rates) along with a safe operation will become even more important. The operational experience from Run-1 and the present plans of upgrade and consolidation are presented along with a discussion from the view point of the detector monitoring and control system.
\end{abstract}

KeYwords: ATLAS, Resistive Plate Chambers, DCS, Trigger, LHC. 


\section{Contents}

1. Introduction [1]

2. The ATLAS Resistive Plate Chambers

3. Detector status and performance during Run-1

母. The RPC Detector Control System 2

5. Detector and DCS operation during Run-1

$5.1 \mathrm{HV}$ operation cycle following the LHC fill 4

5.2 Automatic HV working point correction

6. Detector monitoring and background measurements [5

7. Run-2 preparation 7

7.1 The RPC gas system 8

7.2 Lower sectors chamber upgrade

8. Conclusions 9

\section{Introduction}

During the Run-1 period, from April 2010 till February 2013, the Large Hadron Collider (LHC) has provided proton-proton collisions at a centre-of-mass energy of 7 and $8 \mathrm{TeV}$ with instantaneous luminosities up to $7.73 \times 10^{33} \mathrm{~cm}^{-2} \mathrm{~s}^{-1}$, totaling an integrated luminosity of about $30 \mathrm{fb}^{-1}$. In addition, Run-1 included three periods of about a month of operation with heavy ions: $\mathrm{Pb}-\mathrm{Pb}$ collisions at the ends of 2010 and 2011 and p-Pb in 2013. Since March 2013 the LHC and the related experiments are undergoing a long shutdown (LS-1) during which the accelerator and the detectors are being upgraded in view of Run-2 expected to start in 2015 with proton collisions at a centre-of-mass energy of $13 \mathrm{TeV}$ and higher instantaneous luminosities.

This document illustrates the performance and operational experience of the ATLAS Resistive Plate Chambers (RPCs) during Run-1 focusing on the Detector Control System (DCS), its relevance to the operation and its upgrade and extensions for Run-2. 


\section{The ATLAS Resistive Plate Chambers}

RPCs[1] provide the barrel region of the ATLAS detector [[] with an independent muon trigger and a two-coordinate measurement. The chambers are arranged in three concentric double layers (middle confirm, pivot, outer confirm) covering the pseudo-rapidity range $|\eta|<1.05$ with a detector surface area of about $4000 \mathrm{~m}^{2}$ and are operated in a toroidal magnetic field of about 0.5 Tesla. A transverse momentum low $p_{T}(<10 \mathrm{GeV})$ trigger requires a projective coincidence between hits in the pivot and the middle confirm layer while high $p_{T}$ triggers require hits also in the outer confirm layer. An ATLAS RPC is made of two layers, each with two $2 \mathrm{~mm}$ thick bakelite plates and providing two $\eta$ and two $\phi$ strip planes, where $\eta$ is the pseudo-rapidity and $\phi$ is the azimuthal angle. The chambers are operated with a gas mixture of $\mathrm{C}_{2} \mathrm{H}_{2} \mathrm{~F}_{4}(94.7 \%)$ - iso- $\mathrm{C}_{4} \mathrm{H}_{10}(5 \%)-\mathrm{SF}_{6}(0.3 \%)$ at a relative humidity of about $35 \%$. The high voltage (HV) working point is chosen to be at $9.6 \mathrm{kV}$ at a temperature of $24^{\circ} \mathrm{C}$ and a pressure of $970 \mathrm{mbar}$. In these conditions the RPCs work in saturated avalanche mode inducing, for a minimum ionizing particle, a prompt charge of about $1 \mathrm{pC}$ on the pick-up strips and delivering in the gas an average charge of $30 \mathrm{pC}$. The discharge electrons drift in the gas and the signal, induced on pick-up copper strips, is read out via capacitive coupling, and detected by the front-end electronics. Read-out strips have a typical width of $\sim 30 \mathrm{~mm}$ and are grouped in two $(\eta$ and $\phi)$ read-out panels with strips orthogonal to each other. Custom made front-end electronics amplifies, discriminates and converts the detector signals to Emitter Coupled Logic (ECL) standard. These signals are passed to the on-detector trigger electronics [3] which, by requiring appropriate coincidences in the $\eta$ and $\phi$ detector layers, provide ATLAS with a Level 1 trigger decision along with the detector data for accepted events.

\section{Detector status and performance during Run-1}

Data taking during Run-1 was very successful for the RPC system which has been operated with a fraction of active readout channels of $97 \%$ (of a total of $370 \mathrm{k}$ ) and an average active trigger area of (99.0 99.5)\% [ [G] []. The main reason of inactive channels was the disconnection of the gas gaps from the high voltage due to gas leaks. The impact in terms of coverage and trigger efficiency was anyhow very limited thanks to the built-in redundancy with each detector layer having two layers each for the two-coordinate measurement. The data taking was remarkably stable with around $99.0 \%$ (99.8\% in 2012) of the collected data flagged as good for physics analyses.

\section{The RPC Detector Control System}

The DCS is in charge of safely operating and monitoring all of the detector aspects including the power system delivering the detector high and low voltage supply. The hardware chosen for the power system of the RPCs was based on the commercial CAEN EASY (Embedded Assembly SYstem) solution [G], which consists of magnetic field (up to $0.2 \mathrm{~T}$ ) and radiation tolerant electronics and based on a master-slave architecture. Branch controllers, hosted in a CAEN mainframe, act as master boards, monitoring and controlling electronics in several crates located in the experimental area. More details can be found in [7]. Fig. 1] shows an updated picture of the DCS architecture in preparation of Run-2. In order to guarantee maintainability and support for all of Run-2, 


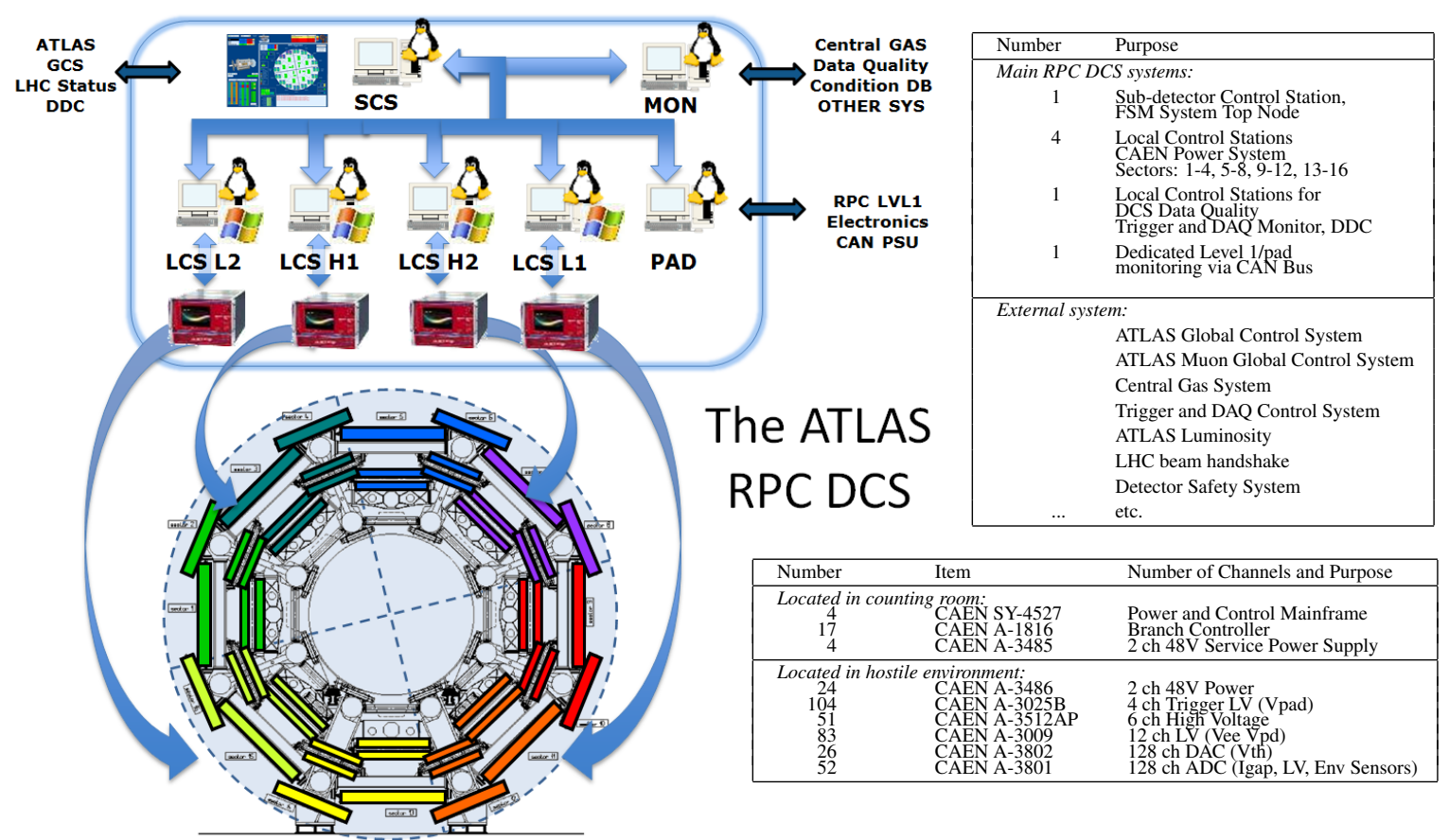

Figure 1. Layout the ATLAS RPC Detector Control System. The infrastructure with the DCS computing hardware and the CAEN mainframes is sketched. In the top table on the right, the computing hardware is listed. Presently the system is composed of 7 machines, 4 Local Control Stations connected, one to one, via OPC, to the CAEN mainframes each monitoring one quadrant of the detector. At the top of the hierarchy, the RPC Sub-detector Control Station supervises the whole system and connects to external systems and to the ATLAS Global Control Station. Additional systems including the Gas System, the Data Quality, and the Level 1 Trigger and DAQ monitoring (DAQ-to-DCS-Communication, DDC) are also shown. For Run-2, the CAEN mainframes and the computers have been upgraded to new hardware and operating system. In the bottom table, the power and monitoring hardware is listed including recently added equipment for new chambers being commissioned for Run-2.

the software platform was upgraded to the new release of the SCADA program now re-branded WinCC-OA[ [8]. The computers and the operative systems were also upgraded. The system is now composed of 7 multi-core rack-mounted PCs all running Scientific Linux. For the communication to the CAEN mainframes (also upgraded from SY-1527 to newer SY-4527) a Windows 2008 Server virtual machine is run on one of the cores of local computers to allow the communication with OPC-DA.

\section{Detector and DCS operation during Run-1}

When designing the RPC DCS, a large number of settings (Digital-to-Analog-Converter (DAC) $\sim 4000$ ) and monitoring (Analog-to-Digital-Coverter ADC 6500) channels had been integrated into the system to optimize the detector performance and allow precise monitoring at the level of the single gas gap $(\sim 3600)$. The remaining ADC channels are used to monitor, with high granularity, the current drawn by the front-end electronics and the RPC gas and environmental sensors 
(temperature, atmospheric pressure, relative humidity and gas flow). The ability to control by tuning thresholds, and monitor the current of each RPC gap has shown to be very powerful during Run-1 both for tracing problems and for fine tuning the detector. This is particularly important as the RPC performance and ageing is strongly affected by the environmental parameters, namely the temperature $(T)$, the atmospheric pressure $(P)$, and the relative humidity. In addition the possibility of studying online the correlations between the currents of adjacent layers, the trigger rate and their dependency with luminosity has proven to be a very effective tool for spotting developing problems.

In 2011 the RPCs benefited from the introduction of several automatic monitoring and control tools to simplify the detector operation and to optimize the data taking conditions. In particular, one improvement was to add to the RPC DCS the full automatic control of the HV settings. These were automatically adjusted to

- follow the different LHC beam phases from injection, stable beams, to the final dump;

- automatically check the individual gas gaps currents, and recalibrate at each end of fill their pedestals;

- compensate for the local changes of the environmental conditions (mainly local temperature and atmospheric pressure);

- provide an online measurement of the cavern background rates and the instantaneous luminosity.

These aspects are briefly described below.

\subsection{HV operation cycle following the LHC fill}

During 2011 automatic actions on the HV settings, dependent on the LHC status were introduced. Besides the automatic ramping at nominal voltage during stable beams, these included the measurement of the gas gap current at the end of the fill, after the beam was dumped. These pedestals allow subtracting precisely, fill by fill, the contribution due to the background from collisions and to provide an estimation of the cavern background and the luminosity itself. Studies have been already presented illustrating the possibility of an independent measurement of the luminosity with

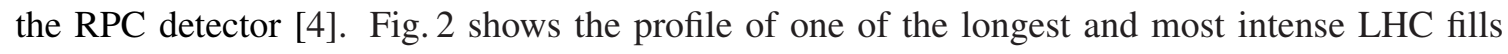
during 2012. The ATLAS luminosity is plotted along with the average current as measured by the RPC DCS. The ratio shows an excellent stability over the whole fill. At the end of the fill, once beam is dumped, effects from detector and cavern activation can be measured. A new calibration point is then taken 20 minutes after beam dump before moving to the standby voltage. A cycle with two standby voltages (6000 and $9000 \mathrm{~V}$ ) was used to allow especially those chambers with reduced gas flow to recover from period with higher current draw.

\subsection{Automatic HV working point correction}

The gas gain, the noise rate and the dark current of an RPC depend on the environmental parameters following the formula:

$$
V_{\mathrm{appl}}=\rho \cdot V_{\mathrm{eff}}=\left(1+\alpha\left(\frac{P-P_{0}}{P_{0}}\right)\right) \cdot\left(1-\beta\left(\frac{T-T_{0}}{T}\right)\right) \cdot V_{\mathrm{eff}},
$$




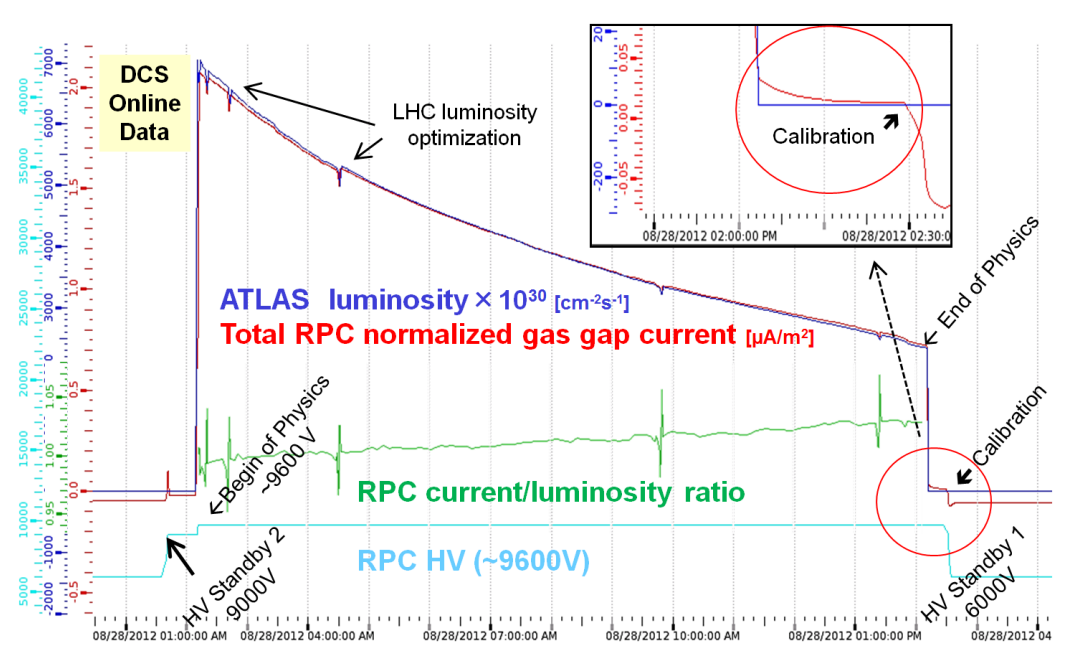

Figure 2. ATLAS instantaneous luminosity and RPC average gap current. The ratio between the two quantities is remarkably constant allowing, once calibrated, for an independent online luminosity measurement and studies on cavern activation background at beam dump. The complex HV cycle with two standby phases and calibration at the end of the fill is also shown.

where $V_{\text {appl }}$ is the applied voltage, $\rho$ is the HV correction factor, $T$ and $P$ are the environment measurements and $T_{0}, P_{0}, V_{\text {eff }}$ are the reference environmental values and $\mathrm{HV}$ settings. The introduced $\alpha$ and $\beta$ factors express the individual correction due to the atmospheric pressure and the temperature, respectively. The atmospheric pressure measurement along with the data of about 300 temperature sensors located on the chambers across the whole system are used to adjust the nearly $280 \mathrm{HV}$ channels of the RPCs. In order to avoid wrong or over-corrected HV set-points, the data from sensors of neighbouring chambers are used only if within a range of validity and then combined. Conservative settings [四 were used: a factor $\alpha=0.8$ for the atmospheric pressure term and $\beta=0.5$ for the temperature. Furthermore, for detector safety, a limit to the HV (9500 V) for chambers with temperature above $26^{\circ} \mathrm{C}$ was set along with independent procedures steadily monitoring the gas flow and the gas gap currents which would lower the corresponding HV set point in case of abnormal readout values. During nominal running, the environmental correction updates every few minutes, refreshing the working points of the HV channels which are adjusted with the lowest ramp up/down speed. The correction is automatically disabled for the periods of no beam or during run set-up. Chambers belonging to different detector areas being at different temperatures can show a spread in the applied voltage of up to $\pm 150 \mathrm{~V}$. In normal operation, the temperature differences remain constant while continuous adjustment follows the trend of atmospheric pressure moving the operating voltage by up to the $3 \%$ of the nominal voltage. From the beginning of 2011, the correction described above has been stably used allowing a general improvement in the detector performance and efficiency[四].

\section{Detector monitoring and background measurements}

The monitored currents and pedestals, the environmental variables and the beam information are used to estimate the average counting rate per surface unit induced by the radiation to study the 
a) Proton-Proton Collisions

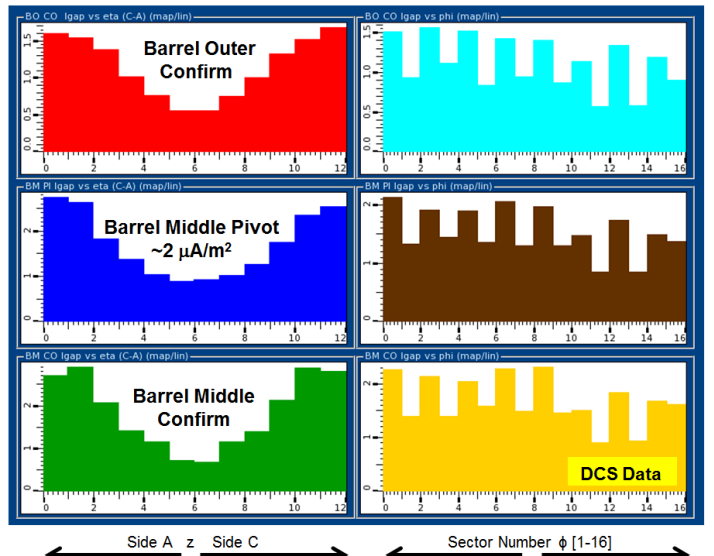

$\leftarrow$ Side $\mathrm{A} z$ Side $\mathrm{C}$ b)

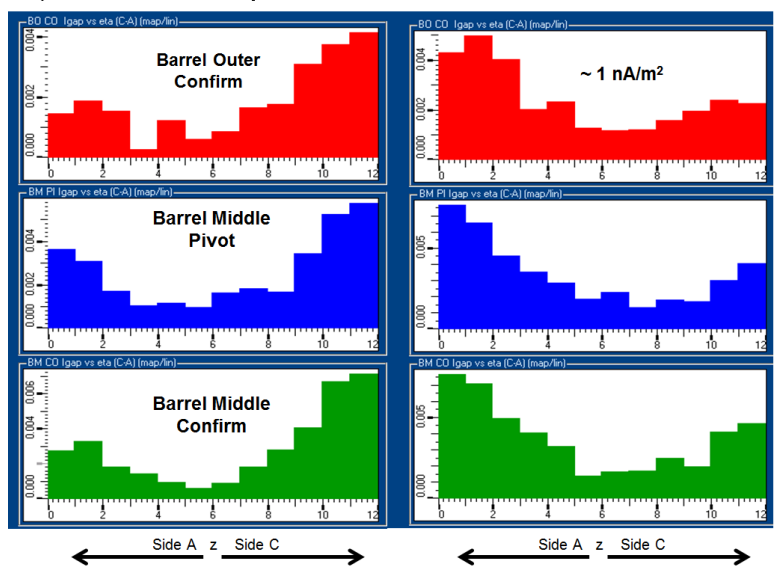

Figure 3. DCS plots displaying the instantaneous pedestal subtracted gas gap currents (per unit area) for the three double layers of RPC chambers. The longitudinal views are shown with bins following the granularity of 12 chambers along $z$ or the 16 sectors along $\phi$. a) Snapshot from p-p collisions taken August $24^{\text {th }} 2012$, 3 a.m., Run 209084, inst. lumi. $4.5 \times 10^{33} \mathrm{~cm}^{-2} \mathrm{~s}^{-1}$ : an average current of $2 \mu \mathrm{A} / \mathrm{m}^{2}$ is measured. Higher currents are seen in the chambers at higher $|z|$. The geometric structure of large/small even/odd sectors is seen along $\phi$ as well as a reduced background in the bottom sectors $(12,13,14)$ due to the shielding of the ATLAS support structure. b) Same plots for heavy ion-proton collisions (Pb-p from January 27 2013 , 3 p.m., Run 218338 , inst. lumi. of $8.6 \times 10^{28} \mathrm{~cm}^{-2} \mathrm{~s}^{-1}$ and p-Pb from February $4^{\text {th }} 2013,7$ p.m., Run 218771 , inst. lumi. of $8.8 \times 10^{28} \mathrm{~cm}^{-2} \mathrm{~s}^{-1}$ ): in this case average currents of $1 \mathrm{nA} / \mathrm{m}^{2}$ are measured and the imbalance along $z$ coming from asymmetric collisions is clearly visible.

beam background and activation effects and their relation with the integrated and instantaneous luminosities. These features are implemented online in the DCS, allowing instantaneous monitoring and publishing of the background distribution. Fig. 3 shows online distributions of the beam induced detector currents as measured and normalized by the detector surface for the three double layers of RPC chambers. Data from three different runs are shown for comparison: proton collisions on the left, lead ions-proton and proton-lead ions on the right. Although the luminosity and the particle background are very much reduced in this second case, the system is able to trace with precision background maps also for these conditions. These data can be used as an independent measurement to study the cavern background and to verify that simulations are compatible. Fig. (4[0] compares the background distributions (scaled at same luminosity) for two runs taken respectively in 2011 and 2012. The comparison highlights two main features: (i) the local decrease of rate of about $20 \%$ between 2011 and 2012, in the bins \pm 4 and \pm 5 , due to a reinforcement of the shielding in correspondence of an existing gap between the end-cap and the barrel; (ii) the significant and systematic excess of rate of about 5\% in most of the other bins which is compatible with the rate increase associated to the energy increase from 7 to $8 \mathrm{TeV}$ and for which the data are not corrected. The same data can also be used to extrapolate the background rates at higher luminosities. Fig. [5] shows the DCS backgrounds for $L=10^{34} \mathrm{~cm}^{-2} \mathrm{~s}^{-1}$ and $14 \mathrm{TeV}$ centre-of-mass energy, in both coordinates, $z$ and $\phi$, converted into trigger rate using the conversion factor of 30 pC per hit. 

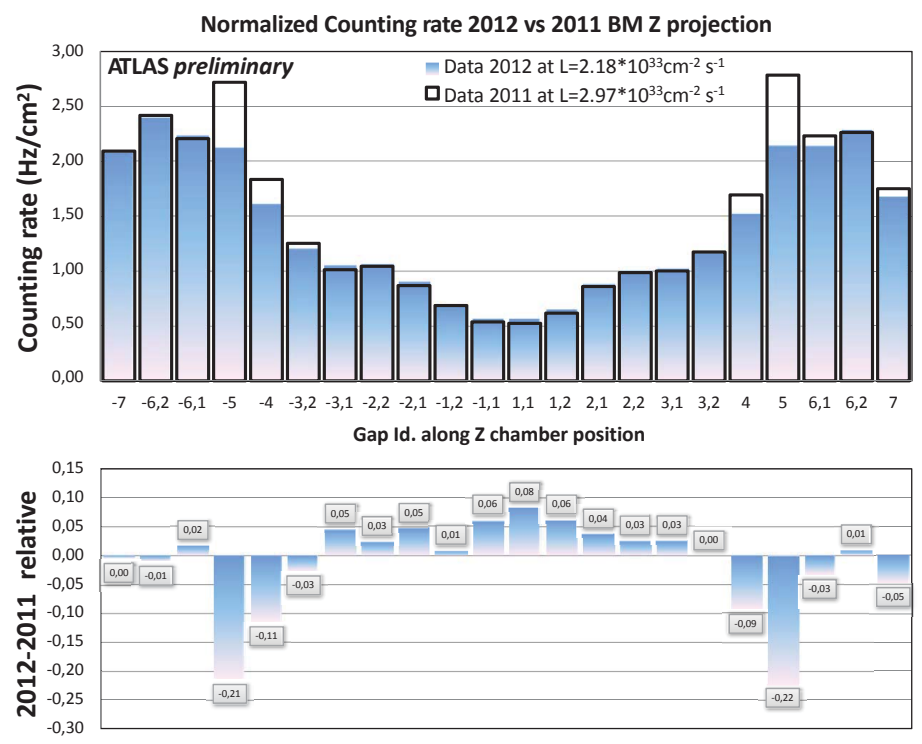

Figure 4. Comparison of counting rate distributions of the RPC barrel middle layers projected on the $z$ coordinate of 2011 data, taken at $L=2.97 \times 10^{33} \mathrm{~cm}^{-2} \mathrm{~s}^{-1}$ at $7 \mathrm{TeV}$, vs. 2012 data, taken at $L=2.18 \times$ $10^{33} \mathrm{~cm}^{-2} \mathrm{~s}^{-1}$ at $8 \mathrm{TeV}$. Both distributions are normalized at $L=10^{33} \mathrm{~cm}^{-2} \mathrm{~s}^{-1}$. Each bin represents a gas gap identified with a progressive number increasing in module with the pseudo-rapidity $\eta$. The lower panel displays the relative difference [Q].

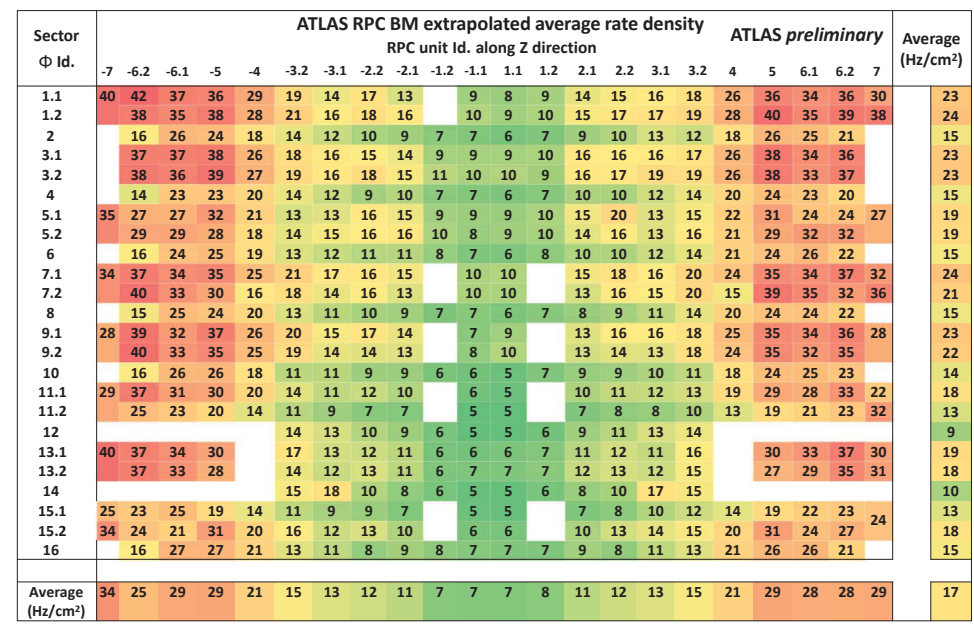

Figure 5. Average counting rate distribution $\left(\mathrm{Hz} / \mathrm{cm}^{2}\right)$ of the RPC barrel middle layers of ATLAS. The rate is derived from instantaneous gap current by applying a conversion factor of $30 \mathrm{pC} /$ count. The current is measured in run 203876 at $L=5.93 \times 10^{33} \mathrm{~cm}^{-2} \mathrm{~s}^{-1}$ at $8 \mathrm{TeV}$. The values displayed are normalized to $L=10^{34} \mathrm{~cm}^{-2} \mathrm{~s}^{-1}$ at $14 \mathrm{TeV}$. The horizontal axis displays the id. of the gas gap along the $z$ coordinate, the vertical axis is the sector and semi sector id covering the azimuthal coordinate [Q].

\section{Run-2 preparation}

The layout of the DCS for Run-2 has been already described. Several additional improvements are on the way in preparation of Run-2 mainly focusing at simplifying the operation, improve the diagnostics and make the alarm reporting stable as a function of the instantaneous luminosity. In 


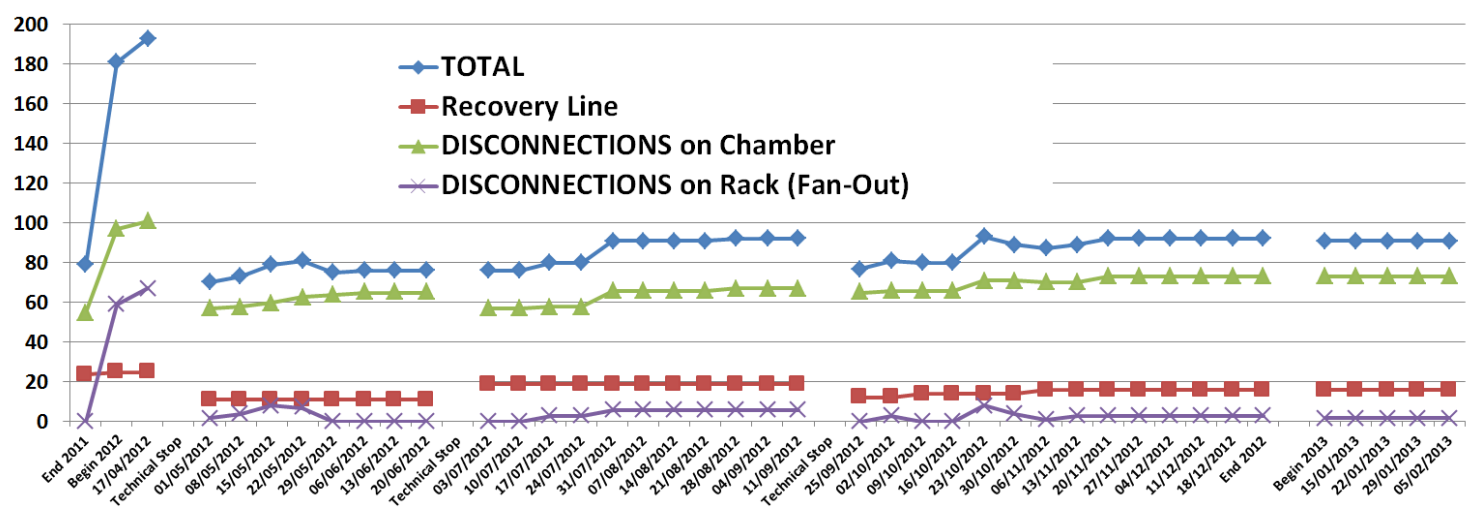

Figure 6. Number of gas gaps disconnected from HV for the period 2012-2013. The number of gas volumes either disconnected or operated at non-nominal conditions has been below $2.5 \%$, with 92 being off due to broken gas inlets or being kept at lower voltage for recovery due to problems caused by insufficient gas refresh rate, giving rise to anomalous operating currents. At begin of 2012 a much larger number of gaps were disconnected due to air and oxygen contamination in the gas system which was solved prior to the begin of data taking.

addition, the RPC system in preparation for Run-2 is undergoing an important upgrade on the gas system and the installation of new chambers in the lower sectors, both briefly discussed below.

\subsection{The RPC gas system}

The ATLAS RPC gas system supplies the detector via a total of 128 individual lines, organized in 5 racks located at different heights to provide the RPC chambers with similar overpressure. The total detector gas volume is approximately $14 \mathrm{~m}^{3}$. The input and output flows of the 128 manifolds are individually monitored both by the central gas system and by the RPC DCS via independent sensors connected to the CAEN ADC boards. The gas is flushed in closed loop: during the operation in 2012, the system was run stably with a total gas flow of approximately $6 \mathrm{~m}^{3}$ per hour and a fresh gas fraction up to $700 \mathrm{l} / \mathrm{h}$. According to design, the chambers were supposed to run at the nominal LHC luminosity of $10^{34} \mathrm{~cm}^{-2} \mathrm{~s}^{-1}$ with one gas volume exchange per hour, i.e. a factor 3 higher than what achieved during Run- 1 . This will be particularly relevant for Run-2 when a luminosity beyond design is expected. One problem which appeared from beginning of operation was the development of leaks due to fragility of the gas inlets when tension from the gas piping is applied. Fig. 6 shows the number of gas gaps disconnected from the HV supply because of a bad gas flow or too high monitored current. The trend has been quite stable during the whole year of operation with a maximum number of 92 gaps either disconnected or operated at lower voltage (out of 3600 , i.e. the $2.5 \%$ ). During the LS-1 a large campaign of gas leak repair has started aiming at the complete recovery of the leaks before the beginning of Run- 2 by using a high density epoxy glue[10]. The central gas system has also been upgraded to allow a higher gas flow, a smoother pressure regulation and a more precise monitoring of the increase of oxygen inside the system. These interventions should allow running at the design gas flow. In addition, as done already during the winter shutdown of 2011[四], a new scheme with individually tuned impedances as function of the chamber volume and the expected integrated radiation will be installed across the whole detector. On the RPC DCS side a higher granularity monitoring system is also being 
commissioned. At each chamber output an additional sensor is added to monitor the output flux. The data from a row of 6 sensors belonging to the same half sector is sent to a multiplexer whose output is readout by the existing ADC channels of the DCS system. This will allow spotting gas leaks in advance and with a much higher accuracy [10] reducing the time required for tracing the affected chambers.

\subsection{Lower sectors chamber upgrade}

One of the main upgrades of the RPC system for Run-2 is the installation and commissioning of new chambers in the bottom sectors $(12,13,14)$. The overall barrel trigger acceptance will be increased by $1 \%$ in sector 13 by instrumenting an area previously left available for detector access. While for the outer plane the usual $2 \mathrm{~mm}$ gap RPCs will be installed, in the barrel middle plane a new generation $1 \mathrm{~mm}$ gap RPCs will be used. In sector 12 and 14, 20 chambers already installed since 2007 but not equipped with the trigger electronics will be commissioned providing an additional increase of $2.8 \%$ in the barrel trigger acceptance. Services and the DCS have been accordingly extended during LS-1 to monitor and control the new chambers.

\section{Conclusions}

The ATLAS RPCs have worked very well during Run-1 delivering good trigger and data for physics. The detector redundancies along with the extensive monitoring capabilities allowed overcoming promptly a few weak points in the infrastructure and providing valuable data in view of the running at higher luminosities. The upgrade and consolidation activities performed during the LHC shutdown and the extensions in the DCS shall further improve the detector operation and performance for Run-2 and beyond.

\section{References}

[1] R. Santonico and R. Cardarelli, "Development of resistive plate counters", Nucl. Instrum. Meth. A 187, 377 (1981)

[2] ATLAS Collaboration, "The ATLAS Experiment at the CERN Large Hadron Collider", 2008 JINST 3 S08003.

[3] F. Anulli et al., "The Level-1 Trigger Muon Barrel System of the ATLAS experiment at CERN", 2009 JINST 4 P04010.

[4] G. Aielli, M. Bindi and A. Polini, on behalf of the ATLAS Collaboration, "Performance, operation and detector studies with the ATLAS Resistive Plate Chambers," 2013 JINST 8 P02020.

[5] D. Boscherini, on behalf of the ATLAS Collaboration, "Performance and operations of the ATLAS RPC system in LHC Run-1", RPC 2014, Tsinghua, Beijing 2014, these proceedings.

[6] CAEN S.p.A. http://www.caen.it

[7] A. Polini [ATLAS Muon Collaboration], "Design and performance of the detector control system of the ATLAS resistive-plate-chamber muon spectrometer," Nucl. Instrum. Meth. A 661, S15 (2012).

[8] ETM professional control GmbH. http://www.etm.at/

[9] ATLAS Collaboration, "RPC Cavern Background Plots". https://atlas.web.cern.ch/Atlas/GROUPS/PHYSICS/MUON/PublicPlots/2014/ATL-COM-MUON-2014-044/

[10] E. Pastori, on behalf of the ATLAS Collaboration, “The ATLAS RPC Gas Distribution”, RPC 2014, Tsinghua, Beijing 2014, these proceedings. 